\title{
Slowing dynamics of a supersonic beam, simulation and experiments
}

\author{
Mehdi Hamamda ${ }^{1}$, Thierry Taillandier-Loize ${ }^{1,2}$, Jacques Baudon ${ }^{1, a}$, Gabriel Dutier ${ }^{1}$, Francisco Perales ${ }^{1}$, \\ and Martial Ducloy ${ }^{1,3}$ \\ ${ }^{1}$ Laboratoire de Physique des Lasers, Universite Paris 13, av. J.B. Clément, 93430 Villetaneuse, France \\ ${ }^{2}$ Institute Mines-Telecom, Telecom Sud-Paris, Department Electronics and Physics, 9 rue Charles Fourier, \\ 91011 Evry Cedex, France \\ ${ }^{3}$ PAP, School of Physical and Mathematical Sciences, Nanyang Technological University, 637371 Singapore
}

Received: 20 May 2015 / Received in final form: 8 July 2015 / Accepted: 9 July 2015

Published online: 14 August 2015 - (C) EDP Sciences 2015

\begin{abstract}
In this paper we present numerical and experimental methods aimed to study the evolution in space and time of a slowed supersonic beam. These generic methods are applicable to a variety of beams and decelerating techniques. The present implemented experimental set up is based upon Zeeman slowing of a metastable atom beam. The detection uses a channel-electron multiplier and a delay-line detector allowing time-of-flight analysis and numerical image reconstruction. In particular a depopulation effect at the centre of the beam is evidenced. In view of quantifying the slowing process, Monte Carlo calculations based on rate-equations are detailed.
\end{abstract}

\section{Introduction}

Mechanical effects induced by light forces, as deflection, focalisation, cooling of atoms by lasers, has become an important and widely studied field, both theoretically $[1,2]$ and experimentally [3-5], in the larger domain of interaction of light with atoms. From the theoretical point of view, in many cases - including the present one - a semiclassical approximation, along the lines defined in reference [6] and elsewhere, is justified, which greatly simplifies the treatment of the external motion since the atom is considered as a point-like particle characterized by its position $\mathbf{r}$ and velocity $\mathbf{v}$. Recently many experiments based on atom - light interactions gained in interest due to the need of slowing and trapping novel species, such as 3D trapping of $\mathrm{Yb}$ Rydberg atoms [7]. A global understanding of the process became necessary to increase slowing efficiencies and trapping rates, especially for heavy atoms of special wavelengths at which low powers are available, e.g., lasers in the $400 \mathrm{~nm}$ range.

Since the seminal work by Phillips and Metcalf [4] followed in particular by Aspect et al. [8], many progresses have been accomplished in the field of slowing atoms. Originally the proposed slowing process - so-called Zeeman slower - was based upon a resonant atom-light interaction within a special magnetic field profile. The first slowing of metastable neon atoms has been

\footnotetext{
a e-mail: jacques.baudon@univ-paris13.fr
}

realized by Shimizu et al. [9] who used basically the same method, with an adapted wavelength. Many papers have been dedicated to the behaviour of an atomic beam during the slowing process, but they all consider a special type of experiment, namely a standard atomic source providing an effusive thermal beam which is then collimated, and further slowed down by a Zeeman slower. Since the work of Blatt et al. [2], numerical simulations have been performed as well as experimental investigations [10-14] with, in most cases, the goal of obtaining a high brilliance slowed beam able to efficiently load a magnetooptical trap. At the time of these previous works, computing capabilities were surely limited compared to what they are nowadays, which has led authors to develop remarkably efficient schemes aimed to handle by many aspects (if not exhaustively) atomic motion in light beams. More recently, light-atom interaction processes have been the subject of detailed investigations $[15,16]$, some of them involving metastable atom beams and using Monte Carlo simulation in theoretical treatments [17].

We describe here a novel method of calculation that can be applied to different types of slowing processes as well as to any type of atom or molecule. In this method, owing to the recent progress in calculus resources, the behaviour of atoms in a beam during the slowing process is analysed step by step, using a Monte Carlo scheme. It will allow us to get calculated 2D images and radial profiles of the slowed beam. Other important characteristics as the coherence radius, the angular aperture, etc. are also 


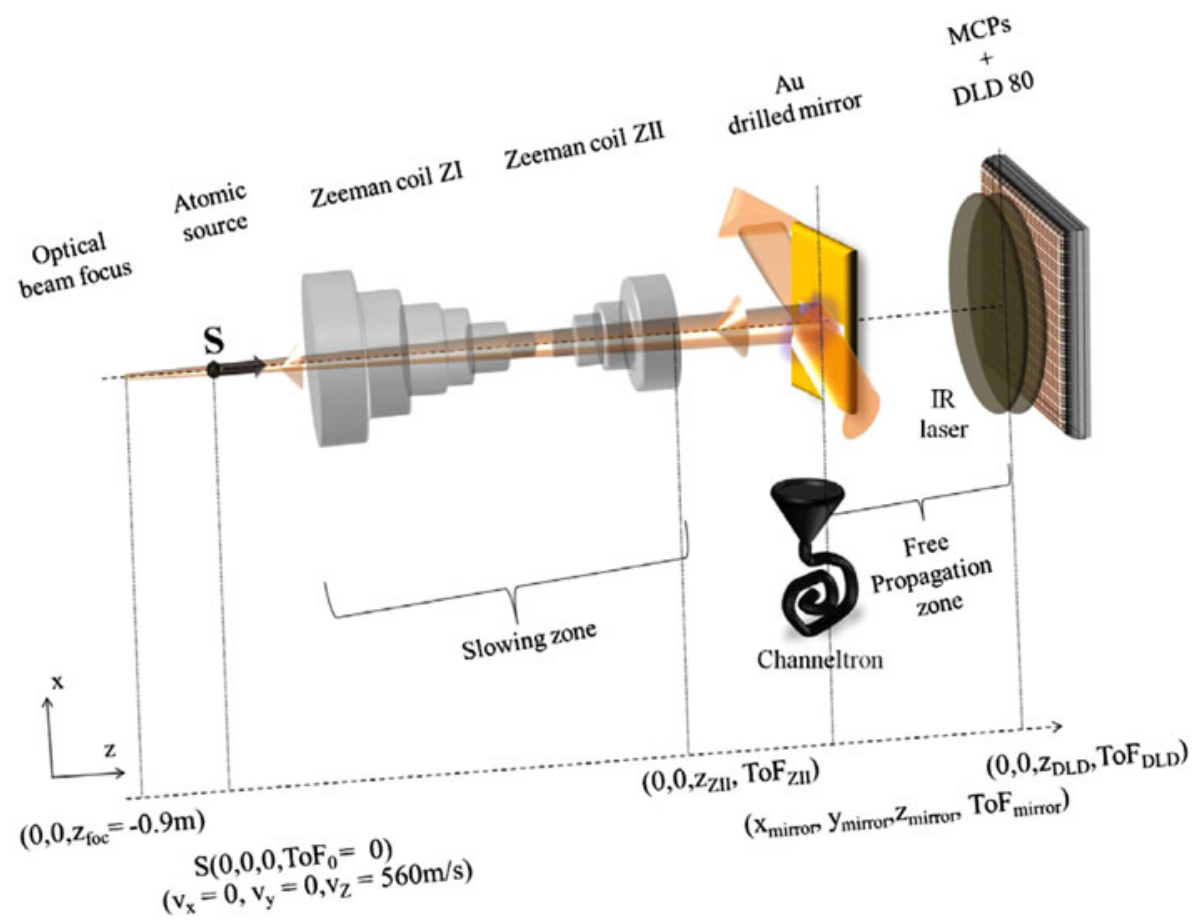

Fig. 1. Principle scheme for the Zeeman slowing of a supersonic Ar* beam. From a point-like source $\mathrm{S}\left(0,0,0, \mathrm{ToF}_{0}\right) \mathrm{Ar}^{*}\left({ }^{3} \mathrm{P}_{2}\right)$ atoms propagate through the first and second Zeeman coils ZI and ZII. The $811.531 \mathrm{~nm}$ resonant laser counter-propagates from the gold mirror to focus point $z_{\mathrm{foc}}$, decelerating the atoms by radiative force. Finally, atoms experience a free-flight zone from the gold mirror coordinate to the delay-line-detector (DLD80). In the lower part, some important points and their spatio-temporal coordinates are given.

available. Since the 3D atomic motion within the laser field is considered, border effects can be studied, while they were not directly accessible by a simple use of the radiative force model.

Experimentally, the use of a double detection, first by a channel-electron multiplier, second by a delay-line detector (DLD80 from Roentdeck) located at a larger distance, allows us to determine the final atom velocity within a few percent accuracy. Moreover the DLD80 provides us with transverse beam profiles that can be readily compared to results of numerical calculations. It is finally shown that the experimental characteristics of the slowed beam are equivalent to the calculated ones, assuming a point-like source, which represents the lowest limit accessible experimentally.

\section{Experimental set-up}

\subsection{Production and deceleration of metastable argon atoms}

The experiment is based upon a high-brilliance supersonic beam of metastable argon atoms $\left(\mathrm{Ar}^{*}{ }^{3} \mathrm{P}_{2}\right)$, with a $10^{-6}$ efficiency $[18,19]$. Thanks to the metastability exchange process [20-22], the resulting flux of Ar* atoms $\left(10^{9}\right.$ atoms $\left.\mathrm{s}^{-1}\right)$ is satisfactory as well as the kinematical properties of the beam (angular width $\delta \theta_{\mathrm{FWHM}}=$ $0.7 \mathrm{mrad}$, velocity dispersion $\delta v / v=5 \%$ ).
The beam deceleration uses a standard Zeeman slower. It consists in a counter propagating, $\sigma+$ polarized infrared laser beam coupled to a special profile of magnetic field aimed to maintain $\mathrm{Ar} *\left({ }^{3} \mathrm{P}_{2}\right)$ atoms at resonance, by a compensation of the local Doppler shift by an appropriate Zeeman shift. The laser system is an extended cavity diode, locked on the ${ }^{3} \mathrm{P}_{2}-{ }^{3} \mathrm{D}_{3}$ transition wavelength $(811.5 \mathrm{~nm})$, and coupled to an amplifier which provides a power of up to $120 \mathrm{~mW}$, i.e., up to $30 \mathrm{~mW}$ being available in the decelerator. The laser frequency is red-detuned by $\delta_{\mathrm{L}}=-340 \mathrm{MHz}$. The light beam is injected in the device by means of a gold mirror the edge of which is an ellipse (minor axis $1 \mathrm{~cm}$ ), positioned in the ultra-highvacuum chamber at an angle of $45^{\circ}$ with respect to the atom beam axis $z$ (see Fig. 1). The local detuning of an atom of velocity $\mathbf{v}$ at position $\mathbf{r}(x, y, z)$ in the laser field and magnetic field $B(z)$ is given by:

$$
\Delta=2 \pi \delta_{\mathrm{L}}-\mathbf{k}_{\mathrm{L}}(\mathbf{r}) \cdot \mathbf{v}(\mathbf{r})+\hbar^{-1}\left(g_{\mathrm{f}} m_{\mathrm{f}}-g_{\mathrm{i}} m_{\mathrm{i}}\right) \mu_{\mathrm{B}} B(z),
$$

$\mathbf{k}_{\mathrm{L}}$ is the laser wave vector, $g_{i, f}$ and $m_{i, f}$ are respectively the initial and final Landé factors and magnetic numbers; $\mu_{\mathrm{B}}$ is the Bohr magneton. The longitudinal magnetic field $B(z)$ is produced by two subsequent coils ZI, ZII. Its profile is shown in Figure 2. The initial velocity is $v_{0}=560 \mathrm{~m} / \mathrm{s}$ along $z$ axis. From the resonance condition $\Delta=0$, the velocity at the end of coil ZI (where $B=0$ ) is readily derived: $v_{I}=275 \mathrm{~m} / \mathrm{s}$. Thanks to the current in coil ZII, the final velocity can be tuned from $v_{I}$ to tens of meter per second. 


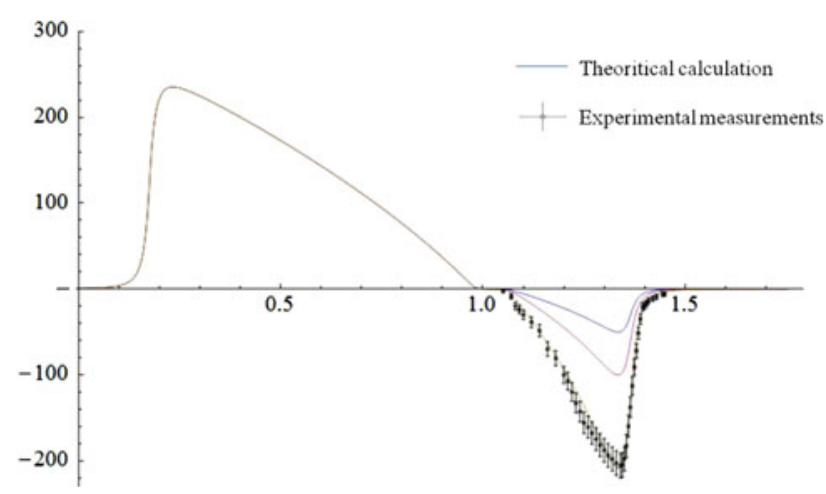

Fig. 2. Magnetic field profile in the Zeeman slower. The adapted profile is chosen to compensate the Doppler shift experienced by the decelerated atoms (see text). The electric current in the first magnetic coil is fixed $I_{\mathrm{ZI}}=300 \mathrm{~mA}$ while it is tunable in the second magnetic coil, $0<I_{\mathrm{ZII}}<1.25 \mathrm{~A}$.

\subsection{Detection schemes}

As explained previously, two different low-flux detectors have been used: (1) a channel-electron multiplier positioned under the golden mirror used as a secondary emission plate. Once synchronized on the starting time of the slowing process $\left(\mathrm{S}\left(0,0,0,0, \mathrm{ToF}_{0}\right)\right.$ in Fig. 1$)$, it allows us to obtain time-of-flight spectra and provides an initialtime reference to the free propagation sequence which follows the slowing sequence; (2) a delay-line detector (DLD80) of diameter $80 \mathrm{~mm}$ [23]. Being synchronized on the same time reference as the channel-electron multiplier, it provides us with a second time-of-flight spectrum. The comparison with the previous one gives the mean final velocity and the temperature in the beam. The time resolution is $400 \mathrm{ps}$. In addition the DLD80 detector gives time-triggered 2D images of the slowed beam, with a resolution of $150 \mu \mathrm{m}$ (FWHM). The low dark noise of this detector ( 0.6 count $\left.\mathrm{cm}^{-2} \mathrm{~s}^{-1}\right)$ allows us observing weak atomic signals. Time-triggered images are easily obtained, using a gate generator and changing the DLD80 imaging driver source code. Figure 3 shows examples of time-triggered images and the related reconstructed radial profiles. The laser power is $30 \mathrm{~mW}$. Figure 3a corresponds to a final velocity of $250 \mathrm{~m} \mathrm{~s}^{-1}\left(\mathrm{ToF}_{\mathrm{det}}=7.75 \mathrm{~ms}\right)$ and Figure $3 \mathrm{~b}$ to a final velocity of $61 \mathrm{~m} \mathrm{~s}^{-1}$, obtained with a current of $900 \mathrm{~mA}$ in coil ZII. The ovoid shadow seen in these images is that of the golden injection mirror. Note that the image naturally appears larger and larger as the velocity decreases, because of the enlargement of the angular aperture of the beam due to spontaneous emission, as it will be seen in Section 3. Figures 3c and 3d show reconstruction of radial profiles at the same velocities, made a posteriori using a $\chi^{2}$ method. At the lowest velocity, a depopulation effect appears at the borders of the shadow, towards the centre of the slowed beam. A complete understanding of this phenomenon relies with the evaluation of the coherence radius, but this latter quantity remains experimentally inaccessible, in so far as it depends on the position and size of the slowed beam effective source and because of the shortness of atomic (a)

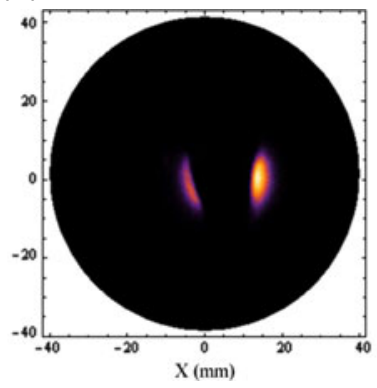

(c)

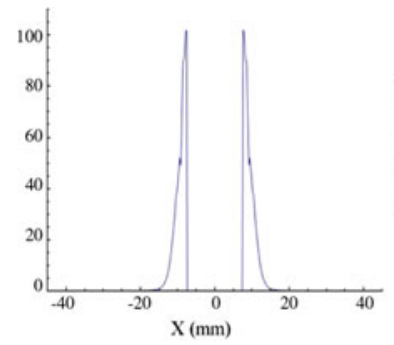

(b)

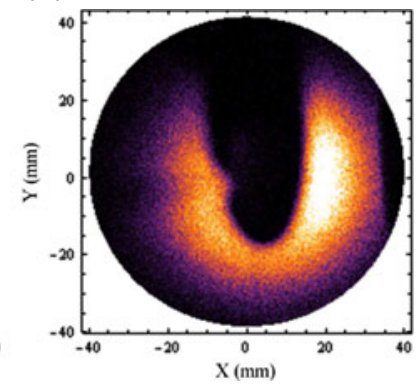

(d)

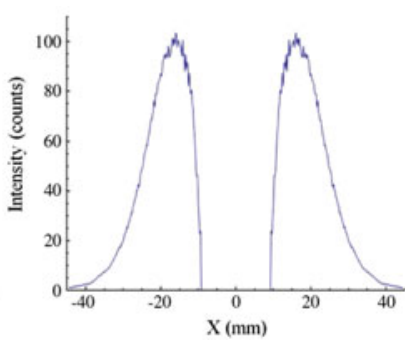

Fig. 3. Triggered images and reconstructed radial profiles (via $\chi^{2}$ method image analysis) for slowed atoms and the corresponding profiles. (a) and (c) $I_{\mathrm{ZII}}=0$ i.e., $v_{\mathrm{F}}=250 \mathrm{~m} / \mathrm{s}$ (b) and (d) $I_{\mathrm{ZII}}=0.9$ A, i.e., $v_{\mathrm{F}}=61 \mathrm{~m} / \mathrm{s}$. In (d) a depopulation phenomenon appears at the centre of the slowed beam starting from $X=17 \mathrm{~mm}$ towards $X=13 \mathrm{~mm}$ and symmetrical position (see text).

wavelengths. This is the interest of carrying out numerical simulations in parallel with experiments.

\section{Numerical simulations}

During the last three decades, Zeeman slower mechanisms have been generally analyzed using in fine the concept of radiative force [24-26]. This force can be expressed as:

$$
\mathbf{F}_{\mathrm{RAD}}=-\frac{1}{2} \hbar \Gamma s_{0}\left[1+s_{0}+(2 \Delta / \Gamma)^{2}\right]^{-1} \mathbf{k}_{\mathrm{L}}
$$

where $\Gamma=2 \pi \times 5.8 \mathrm{MHz}$ is the transition rate, $\Delta(z)$ is the local detuning defined above and $s_{0}(\mathbf{r})=I(\mathbf{r}) / I_{0}$ is the saturation parameter, $I(\mathbf{r})$ being the laser intensity at point $\mathbf{r}$ and $I_{0}=1.4 \mathrm{~mW} \mathrm{~cm}^{-2}$ the saturation intensity. As this force only expresses an averaged effect, it is of little use in a detailed exploration of effects (especially edge effects) experienced by atoms travelling within a laser beam. A more efficient - but somewhat heavier method is to digitize the atomic motion via a Monte Carlo type code (noted MC-code) as a series of "absorption spontaneous emission" cycles. Each cycle includes an absorption time $t_{\mathrm{abs}}$, i.e., the duration of free propagation at some velocity $\mathbf{v}_{i}$ prior to the absorption of a first photon, and an emission time $t_{\mathrm{em}}$, i.e., the free propagation time prior a photon be spontaneously emitted by the atom (in upper state ${ }^{3} \mathrm{D}_{3}$ ) (see Fig. 4). Then the MC-code simulates the behaviour of an individual atom, without any 


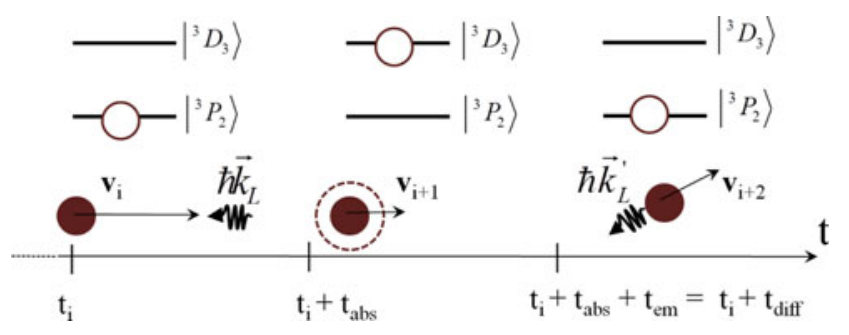

Fig. 4. Model of the atomic motion as a succession of "absorption-spontaneous emission" cycles. The first absorbed photon that will be reemitted by spontaneous emission after a time $t_{\mathrm{abs}}$ (see text). $t_{\mathrm{em}}$ is the free propagation time preceding the spontaneous emission of a photon; $t_{\mathrm{diff}}=t_{\mathrm{abs}}+t_{\mathrm{em}}$ is the diffusion time for a complete sequence.

introduction of collective or averaging effects. It may be noted that actually such random atom-photon exchanges have been previously considered by Vredenbregt and van Leeuwen [27] who, on another hand, almost totally ignored experimental details. The momentum conservation rule implies a velocity change (in fact a decrease) each time a photon is absorbed. It is given by:

$\mathbf{v}_{i+1}=\mathbf{v}_{i}+\mathbf{v}_{\text {rec }}$ with $\mathbf{v}_{\text {rec }}=m_{\mathrm{Ar}}^{-1} \hbar \mathbf{k}_{\mathrm{L}}$ the recoil velocity. $m_{\mathrm{Ar}}$ is the atom mass. In our case $v_{\text {rec }}=1.23 \mathrm{~cm} \mathrm{~s}^{-1}$. After some random time $t_{\mathrm{em}}$, the atom, emitting a photon, decays to its lower level ${ }^{3} \mathrm{P}_{2}$, experiencing a second velocity change given by:

$$
\mathbf{v}_{i+2}=\mathbf{v}_{i+1}+\mathbf{v}_{\text {rand }} \text { with } \mathbf{v}_{\text {rand }}=v_{\text {rec }} \hat{\mathbf{u}}_{\text {rand }},
$$

where $\hat{\mathbf{u}}_{\text {rand }}$ is a normalized random vector whose distribution reproduces the radiation diagram of spontaneous emission. At this point, we finally introduced two random variables $t_{\mathrm{abs}}, t_{\mathrm{em}}$ plus a random vector $\hat{\mathbf{u}}_{\mathrm{rand}}$, the probability densities of which have to be determined.

\subsection{Free propagation before absorption}

A major point in the present MC-code relies on the determination of the $t_{\text {abs }}$ probability density. Indeed cycles as [absorption + induced emission], the period of which only depends on laser intensity, have in general mechanical effects on atom trajectories (optical dipolar or intensitygradient forces). Possible effects of dipolar forces under the present experimental conditions have been examined. The most critical place is located at $3 \mathrm{~cm}$ before the entrance of the Zeeman slower where the red detuning is large $(-340 \mathrm{MHz})$ and the intensity gradient is the most important (there is the focus point of the laser beam, with a waist $w \approx 0.25 \mathrm{~mm}$ ). Nevertheless the magnitude of the radial (focussing) dipolar force does not exceed $4 \%$ of the longitudinal "Zeeman" force. Its effect on fast atom $(560 \mathrm{~m} / \mathrm{s})$ over this short path is negligible, the trajectory being shifted by no more than $1.15 \mu \mathrm{m}$ towards the beam axis.
In principle the $t_{\text {abs }}$ probability density can be derived from the so-called "diffusion rate" (see [28], p. 25):

$$
\Gamma_{\mathrm{diff}}=-\frac{1}{2} \Gamma s_{0}\left[1+s_{0}+(2 \Delta / \Gamma)^{2}\right]^{-1} .
$$

It corresponds to the ensemble [absorption + spontaneous emission], to which is associated the random variable $t_{\mathrm{diff}}=t_{\mathrm{abs}}+t_{\mathrm{em}}$. Its probability density is (taking in account that $\left.t_{\text {diff }} \geq 0\right)$ :

$$
\rho_{\text {diff }}(\xi)=\Theta(\xi) \Gamma_{\text {diff }}^{-1} \exp \left(-\Gamma_{\text {diff }}^{-1} \xi\right)
$$

here $\xi$ is a possible value of the random $t_{\text {diff }}$ and $\Theta$ is the Heaviside function. Actually, in this game, the present choice we just made of $t_{\text {diff }}$ as a "master variable" is arbitrary.

The probability density of $t_{\mathrm{em}}$, under conditions $t_{\mathrm{diff}} \geq$ $t_{\mathrm{em}} \geq 0$, can be written as:

$$
\begin{aligned}
\rho_{\mathrm{em}}(\xi, \zeta)= & \Theta(\xi-\zeta) \Theta(\zeta) \Gamma^{-1} \\
& \times\left[1-\exp \left(-\Gamma^{-1} \xi\right)\right]^{-1} \exp \left(-\Gamma^{-1} \zeta\right)
\end{aligned}
$$

where $\zeta$ is a possible value of $t_{\mathrm{em}}$. The factor $\left[1-\exp \left(-\Gamma^{-1} \xi\right)\right]^{-1}$ in equation (4) is a normalizing factor such that, as needed, for any positive value of $\xi$, one has: $\int_{0}^{\xi} \rho_{\mathrm{em}}(\xi, \zeta) d \zeta=1$.

Actually distributions of the type (3) and (4) imply a Poisson distribution of events in a given interval of time. To test the validity of this implicit assumption we have evaluated the so-called Q-Mandel's parameter, previously considered by Cook [29]. In this evaluation, typical values of variables involved in $Q$ have been taken, namely $\beta=2.62 \mathrm{MHz}$ (half the Einstein coefficient $A$ for the ${ }^{3} \mathrm{P}_{2}-{ }^{3} \mathrm{D}_{3}$ transition in argon), $\Omega$ the Rabi frequency, here about $0.8 \mathrm{MHz}$; the detuning $\Delta$ is much smaller than $\beta$. Under such conditions, we find $Q=-0.127$. In absolute value $Q$ is sufficiently small compared to 1 to reasonably assume an almost pure Poisson distribution.

It is readily seen that, because of the factor $\Theta(\xi-\zeta)$ in equation (4), random variables $t_{\mathrm{diff}}$ and $t_{\mathrm{em}}$ are not independent. As a consequence, the probability density $\rho_{\text {abs }}$ of $t_{\text {abs }}$ cannot be obtained using a simple convolution. To a given couple of values $\xi, \zeta$ of random variables $t_{\text {diff }}$, $t_{\mathrm{em}}$ corresponds one point in plane $(\xi, \zeta)$. The probability to strictly get this point is zero, but it is no longer the case for getting a point within an elementary surface of area $\mathrm{d} S$ containing this point, for which the probability is $\rho_{\text {diff }}(\xi) \rho_{\text {em }}(\zeta) \mathrm{d} S$. The question now is: what is the probability to find the random variable $t_{\mathrm{abs}}=t_{\mathrm{diff}}-t_{\mathrm{em}}$ within a given small interval $[\eta, \eta+d \eta]$ ? This probability has the form $d \eta \rho_{\text {abs }}(\eta)$, where $\rho_{\text {abs }}(\eta)$ is the researched probability density of $t_{\text {abs }}$. The domain $\Delta$ in plane $(\xi, \zeta)$, which corresponds to condition $\eta \leq t_{\text {abs }} \leq \eta+d \eta$, is a narrow strip limited by two lines parallel to the bissextile line, 
(a)

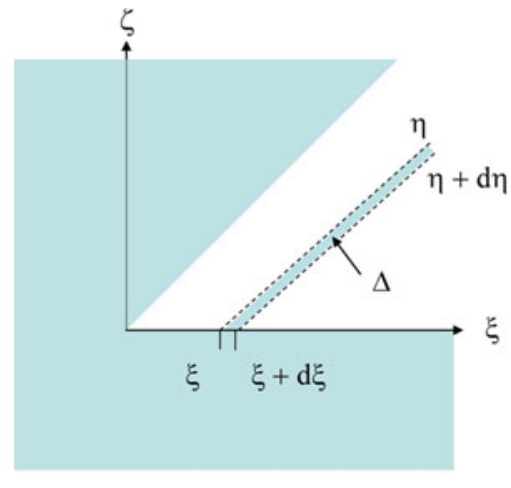

(b)

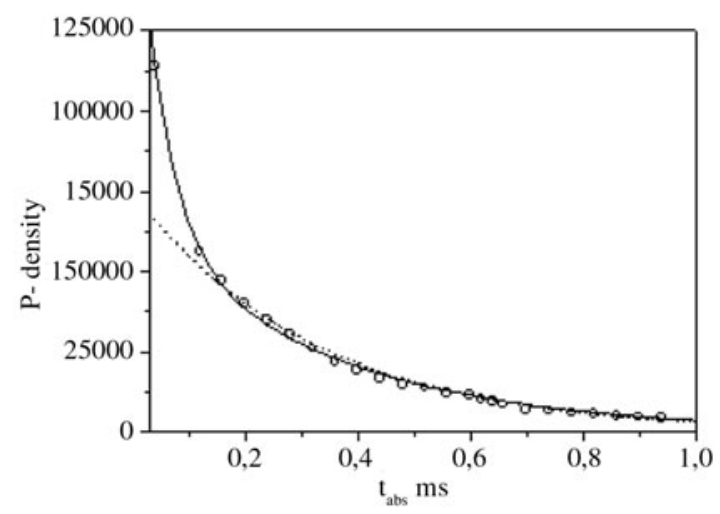

Fig. 5. (a) Domain of variation of $x$ and $z$ and domain $\Delta$ for an infinitesimal variation of $\eta$ (see text), (b) probability density of random variable $t_{\text {abs }}$. Full line: from equation (5a); dashed line: from equation (5b); open circles: from direct statistical simulation (see text).

crossing the $\xi$ axis at abscissas $\eta, \eta+d \eta$ (see Fig. 5a). One readily gets:

$$
\begin{aligned}
d \eta \rho_{\mathrm{abs}}(\eta) & =\iint_{\Delta} \rho_{\mathrm{diff}}(\xi) \rho_{\mathrm{em}}(\xi, \zeta) \mathrm{d} \xi \mathrm{d} \zeta \\
& =\int_{\eta}^{\infty} d \xi \rho_{\mathrm{diff}}(\xi) \int_{\xi-\eta}^{\xi-(\eta+d \eta)} d \zeta \rho_{\mathrm{em}}(\xi, \zeta) \\
& =d \eta \int_{\eta}^{\infty} d \xi \rho_{\mathrm{diff}}(\xi) \rho_{\mathrm{em}}(\xi, \xi-\eta) \\
& =d \eta \frac{1}{2} \Gamma_{\mathrm{diff}} \Gamma \exp \left(-\Gamma_{\mathrm{diff}} \eta\right) \\
& \times{ }_{2} F_{1}\left[\frac{\Gamma_{\text {diff }}+\Gamma}{\Gamma}, 1,1+\frac{\Gamma_{\text {diff }}+\Gamma}{\Gamma}, \exp (-\Gamma \eta)\right]
\end{aligned}
$$

where ${ }_{2} \mathrm{~F}_{1}(a, b, c, u)$ is the hyper geometric function. Figure $5 \mathrm{~b}$ shows this probability density compared to numerical results obtained with diffusion times randomly generated over $10^{6}$ iterations, from which independent randomly generated emission times are subtracted. Also shown is a simplified expression of $\rho_{\text {abs }}$, easier to use in the MC-code, namely:

$$
\rho_{\text {abs }}(\eta) \approx\left(\Gamma_{\text {diff }}^{-1}-\Gamma^{-1}\right) \exp \left[-\left(\Gamma_{\text {diff }}^{-1}-\Gamma^{-1}\right) \eta\right] .
$$

It under-estimates the probability of the lowest values of $t_{\mathrm{abs}}$, which is of little importance since $\left\langle t_{\mathrm{abs}}\right\rangle$ rapidly departs from low values. For sake of simplicity and shorter computing times, the forthcoming MC-code will use expression (5b). Random values of $t_{\mathrm{abs}}$ are generated by:

$$
t_{\mathrm{abs}}=-\left(\Gamma_{\mathrm{diff}}^{-1}-\Gamma^{-1}\right) \log [\operatorname{Rn}[\{0,1\}]],
$$

where $R n[\{a, b\}]$ is a random number uniformly distributed over interval $\{a, b\}$.

At time $t_{i+1}=t_{i}+t_{\text {abs }}$ (see Fig. 4), the atom velocity becomes:

$$
\mathbf{v}_{i+1}=\sum_{\alpha=x, y, z}\left[\left(\mathbf{v}_{i}+\hbar M_{\mathrm{Ar}}^{-1} \mathbf{k}_{\mathrm{L}}\right) \cdot \hat{\mathbf{e}}_{\alpha}\right] \hat{\mathbf{e}}_{\alpha} .
$$

The light wave-vector $\mathbf{k}_{\mathrm{L}}$ at position $\mathbf{r}$ is derived from the definition of equal phase surfaces in a Gaussian laser beam focussed at point $\left(0,0, z_{\mathrm{foc}}\right)$. Setting $z^{\prime}=z-z_{\mathrm{foc}}$, $\Xi=\pi w_{0}^{2} / \lambda$ (co focal parameter, $w_{0}$ being the minimal waist of the laser beam), and $F^{2}=z^{\prime 2}+\Xi^{2}$, one gets:

$$
\mathbf{k}_{\mathrm{L}}(\mathbf{r})=k\left(\begin{array}{c}
-F^{-2} z^{\prime} x \\
-F^{-2} z^{\prime} y \\
-1-F^{-2} \Xi / k-\frac{1}{2}\left(x^{2}+y^{2}\right) F^{-4}\left(\Xi^{2}-z^{\prime 2}\right)
\end{array}\right) .
$$

\subsection{Spontaneous emission}

The propagation of the atom in upper state ${ }^{3} \mathrm{D}_{3}$ is free for a random time $t_{\mathrm{em}}$ generated similarly as $t_{\mathrm{abs}}$ (Eq. (6)) by:

$$
t_{\mathrm{em}}=-\Gamma^{-1} \log [\operatorname{Rn}[\{0,1\}]] .
$$

Afterwards the atom decays to its lower level ${ }^{3} \mathrm{P}_{2}$, spontaneously emitting a photon. As the laser light is polarized $\sigma+$, one can show $[28,30]$ that the velocity vector after this emission is:

$$
\mathbf{v}_{i+2}=\mathbf{v}_{i+1}+\hbar M_{\mathrm{Ar}}^{-1} k \hat{\mathbf{u}}_{\mathrm{em}},
$$

$\hat{\mathbf{u}}_{\mathrm{em}}$ is a random unit vector whose polar angles $\theta$ and $\phi$ are given by:

$$
\theta=\frac{-1+\left(2 \mu+\sqrt{1+4 \mu^{2}}\right)^{2 / 3}}{\left(2 \mu+\sqrt{1+4 \mu^{2}}\right)^{1 / 3}} \text { and } \phi=2 \pi \nu,
$$

with $\mu=\operatorname{Rn}[\{-1,1\}]$ and $\nu=\operatorname{Rn}[\{0,1\}]$.

\subsection{D numerical imaging, characterization of the slowed beam}

Important by-products of Monte Carlo simulation are (i) a $2 \mathrm{D}$ imaging of the slowed beam, in plane $\left(\hat{\mathbf{e}}_{x}, \hat{\mathbf{e}}_{z}\right)$, 
(a)

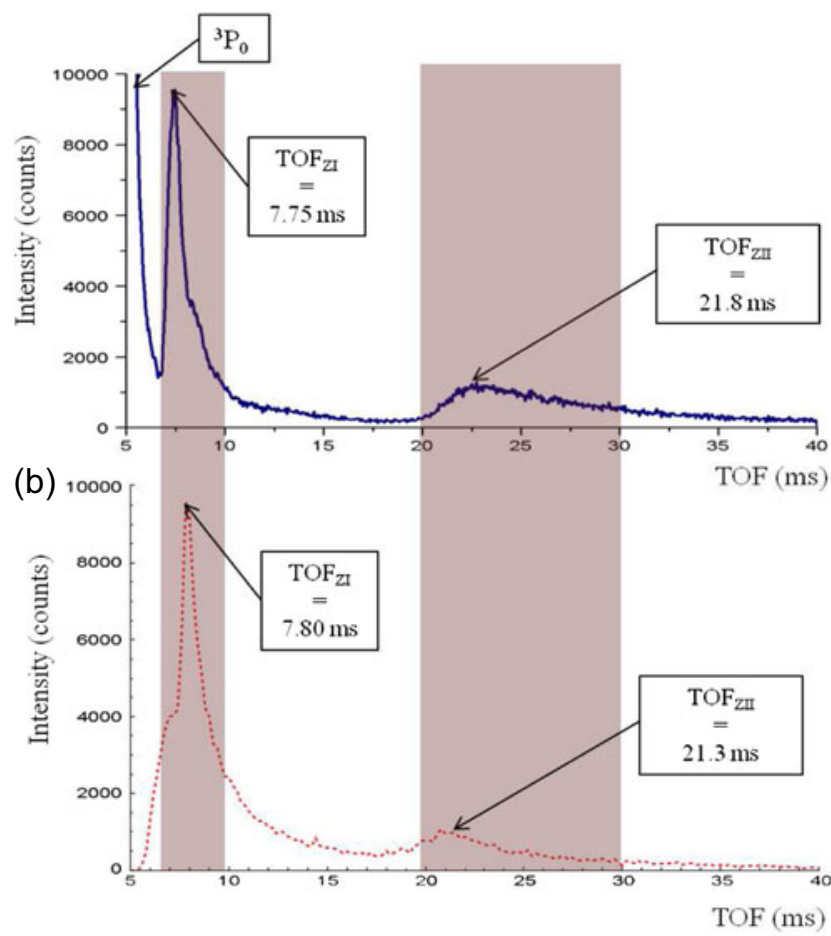

Fig. 6. Temporal structure of the slowed Ar_beam. We compare experimental (a) and numerical (b) spectra obtained for a current $I_{\mathrm{ZII}}=0.9 \mathrm{~A}$ in the second magnetic coil ZII and an effective laser power of $30 \mathrm{~mW}$. In (b), ${ }^{3} \mathrm{P}_{0}$ atoms that are non-sensitive to the slowing process are not shown. The tinted (grey) zones in the two spectra show the good matching (typically $5 \%$ ) between experimental and calculated data.

which gives both position and size of the effective source; (ii) time of flight (ToF) spectra, the comparison of which with experimental data tests the validity of our theoretical approach (Sect. 3.1). Figure 6 presents such a comparison of an experimental spectrum (upper part) acquired using the DLD detector and the corresponding calculated one (lower part). It is seen that a structure appears in both spectra (peak at $\mathrm{ToF}=7.75 \mathrm{~ms}$ and $7.65 \mathrm{~ms}$ respectively in experiment and simulation). It represents atoms decelerated by the first Zeeman coil (ZI) but not captured by the second one (ZII) because they move outside of the laser beam. Indeed a simulation using a laser beam of a twice larger diameter shows that under this condition all atoms are transferred at $\mathrm{ToF}=20.4 \mathrm{~ms}$.

The 2D-imaging process consists of a collection of $5 \times 10^{3}$ atoms displayed over $10^{6}$ positions. The free propagation zone located in between the two detectors (see Fig. 1) is $0.625 \mathrm{~m}$ long. Using the ToF spectra generated by these two detectors, one can determine the final velocity $v_{\mathrm{F}}$ with an accuracy of $10 \%$. Figure 7 shows calculated and observed evolutions of this velocity with respect to the current $I_{\mathrm{ZII}}$ in coil ZII. The discrepancy between calculated and measured values never exceeds $5 \%$. As expected $v_{\mathrm{F}}$ is a linear function of $I_{\mathrm{ZII}}$.

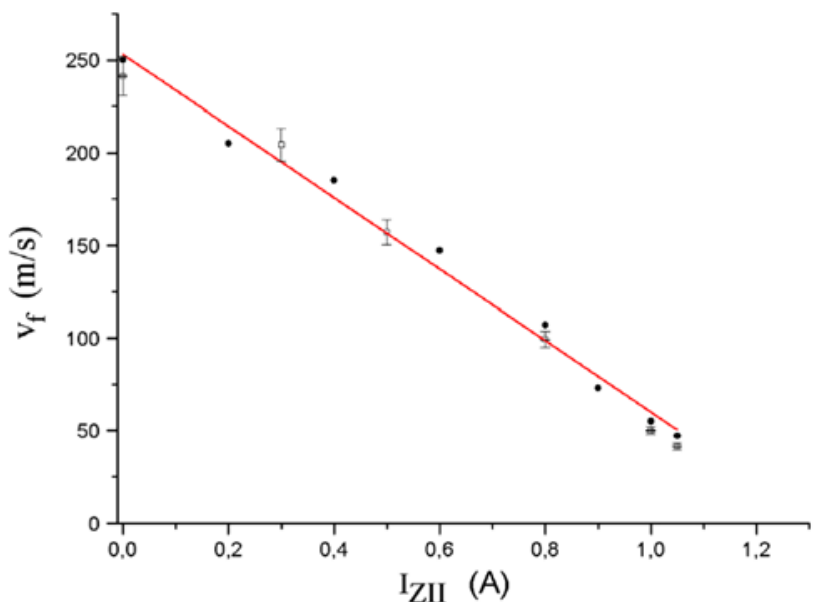

Fig. 7. Evolution of calculated and measured final velocity with respect to the current $I_{\mathrm{ZII}}$ in coil ZII. Black filled circles represent numerical data and open circles with error bars represent the experimental points.

Figure 8 illustrates another possible issue of our MC simulation, namely the lateral profile imaging of slowed beams, in (a) for $v_{\mathrm{F}}=250 \mathrm{~m} / \mathrm{s}$, in (b) for $v_{\mathrm{F}}=61 \mathrm{~m} / \mathrm{s}$. Coherence parameters and angular aperture $\Delta \theta$ are then readily accessible (see Tab. 1). In general, the coherence radius $R_{\mathrm{c}}$ of an atom source of size $a_{\mathrm{S}}$ at a distance $D_{\mathrm{S}}$ from a pair of Young slits is defined as the largest distance between slits for which observable interferences are seen, i.e., $R_{\mathrm{c}}=0.257 \lambda_{\text {at }} D_{\mathrm{S}} / a_{\mathrm{S}}$ where $\lambda_{\text {at }}$ is the de Broglie wavelength [31]. Experimentally $R_{\mathrm{c}}$ is difficult to measure especially because of the shortness of $\lambda_{\text {at }}$, while such a measurement has been made in the case of a thermal supersonic beam [32].

The abrupt increase of $\Delta \theta$ at velocities lower than $150 \mathrm{~m} / \mathrm{s}$ practically forbids the use of such slowed beams in scattering or interferometry experiments, except if a strong collimation and/or focalisation are made. Correlatively, the coherence radius dramatically decreases (Fig. 9), which is a consequence of the large number (20000 to 40000) of spontaneous emissions - and the related random components added to its velocity experienced by a slowed atom. This finally leads to a quasi total loss of coherence.

\subsection{Time-triggered imaging}

In this section experimental and simulated time-triggered images are compared. Numerical imaging is simply realized by backing-up atom parameters (position $\mathbf{r}(x, y, z)$, velocity $\mathbf{v}\left(v_{x}, v_{y}, v_{z}\right)$, time-of-flight $\mathrm{ToF}$, number $N_{\mathrm{ph}}$ of absorbed photons) and plot their spatial density, for the $5 \times 10^{6}$ atoms involved in the calculation. This numerical treatment provides us with spatial profiles, in $\mathbf{r}$, velocity distributions, in $\mathbf{v}$, time-of-flight spectra and absorbed photon density. Four important positions along the $z$ axis are examined, namely the end of coil $\mathrm{ZI}$ at $z_{\mathrm{I}}$, the end of 
(a)

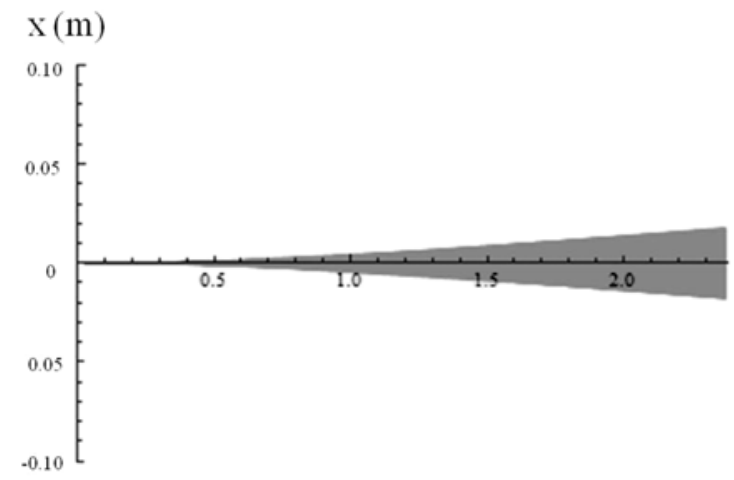

(b)

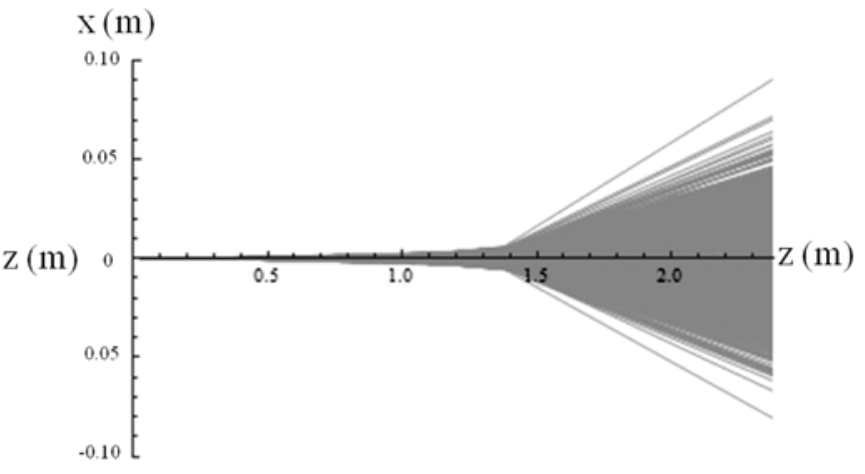

Fig. 8. $2 \mathrm{D}$ imaging of the slowed Ar* supersonic beam for two final velocities: (a) $v_{\mathrm{F}}=250 \mathrm{~m} / \mathrm{s}(\mathrm{b}) v_{\mathrm{F}}=61 \mathrm{~m} / \mathrm{s}$. From these images, one can easily extract the beam divergence and the coherence radius with respect to the position and size of the effective source (see text).

Table 1. Evolution of coherence parameters as functions of the final velocity $v_{\mathrm{F}}: a_{\mathrm{S}}$ is the effective source radius (HWHM), $D_{\mathrm{S}}$ is its distance to the origin position of ToF measurements, $R_{\mathrm{c}}$ is the mean value of the coherence radius, $\Delta \theta$ is the angular aperture. The general relative uncertainty is about $0.3 \%$.

\begin{tabular}{ccccc}
\hline$v_{\mathrm{F}}(\mathrm{m} / \mathrm{s})$ & $D_{\mathrm{S}}(\mathrm{m})$ & $a_{\mathrm{S}}(\mathrm{mm})$ & $R_{\mathrm{c}}(\mathrm{nm})$ & $\Delta \theta(\mathrm{mrad})$ \\
\hline 250 & 0.31 & 0.21 & 490 & 12 \\
205 & 0.68 & 0.79 & 130 & 12 \\
185 & 0.83 & 1.0 & 102 & 15.5 \\
147 & 0.97 & 1.3 & 97.5 & 17 \\
107 & 1.3 & 2.0 & 61 & 32 \\
60 & 1.3 & 3.5 & 60 & 48 \\
47 & 1.35 & 4.7 & 57 & 98 \\
\hline
\end{tabular}

coil ZII at $z_{\mathrm{II}}$, the golden mirror at $z_{\text {mir }}$, the DLD detector at $z_{\text {det }}$.

Figure 10 shows the experimental radial profile of the $61 \mathrm{~m} / \mathrm{s}$ - slowed beam (from Fig. 3d) together with the numerical simulation, for a laser power $P_{\mathrm{L}}=30 \mathrm{~mW}$, a beam diameter $d_{\mathrm{L}}=8 \mathrm{~mm}$ at $z_{\text {mir }}$, focussing at $z_{\mathrm{foc}}=$ $0.9 \mathrm{~m}$. It is seen that, except at the centre (mirror shadow) the agreement is excellent, within an uncertainty of $5.3 \%$ which can be explained by: (i) an uncertainty of $3 \%$ on the distance chopper-detector, (ii) the modelling of the mirror by an infinite plane, ignoring possible distortion coming from the lateral part of the laser beam (see Fig. 1), (iii) the assumption of a perfect alignment of atom and light beams, (iv) the assumption that atoms are initially emitted from a point-like source.

This very good agreement obtained for both wings of the profile makes us confident about the reality of the depopulation effect given by the simulation at the centre and already suggested by experimental data (see Sect. 2.2 and Fig. 3d). This depopulation is estimated to be $10 \%$ of the initial flux and $35 \%$ of the flux of atoms actually captured and slowed down by coil ZII. It is essentially due to laser pressure which is especially important at the centre. Indeed, for fully decelerated atoms, that permanently

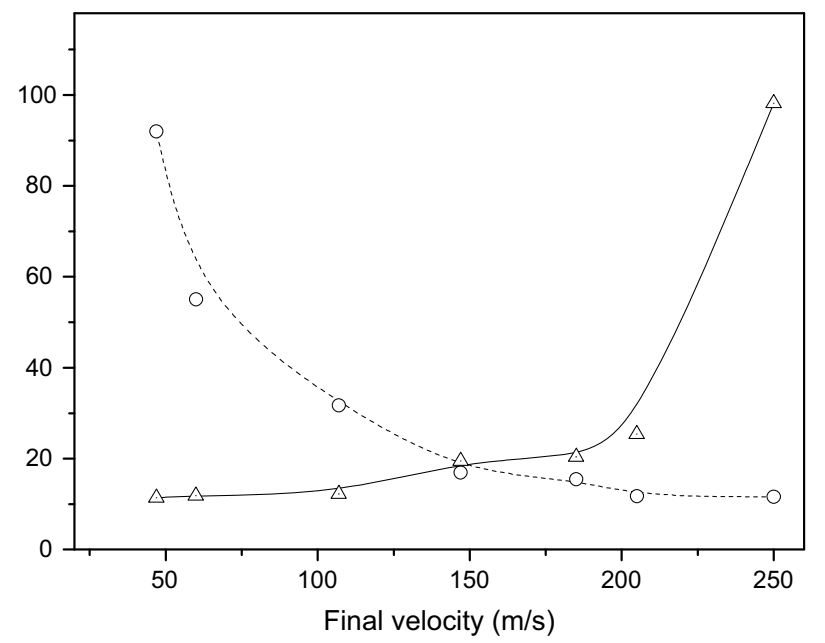

Fig. 9. Dashed line, open circles: angular beam aperture $\Delta \theta$ in mrad; full line, open triangles: coherence radius $R_{\mathrm{c}}$ divided by 5 , in $\mathrm{nm}$.

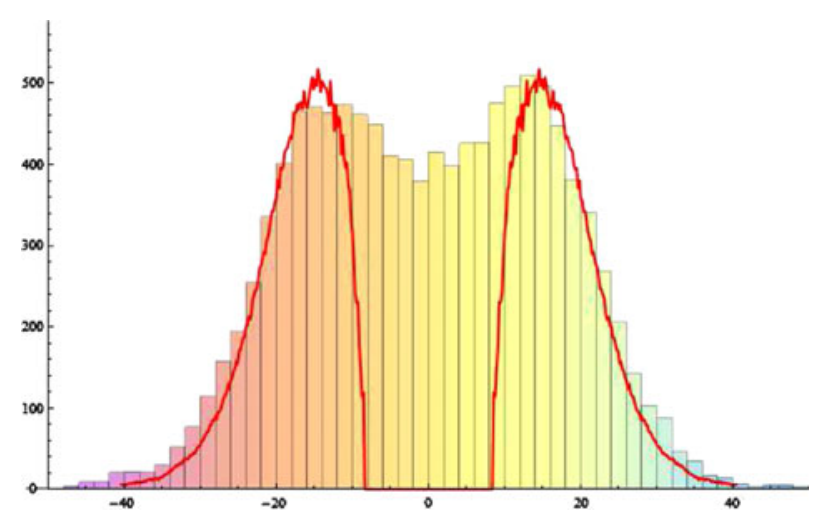

Fig. 10. Superposition of the $61 \mathrm{~m} / \mathrm{s}$ slowed beam radial profile (bold red line) (cf. Fig. 3d) and numerical profile calculated using the MC-code. The agreement between theory and experience in the wings represents $5.3 \%$ accuracy, and the depopulation ratio is estimated to be $10 \%$ (see text). 
(a)

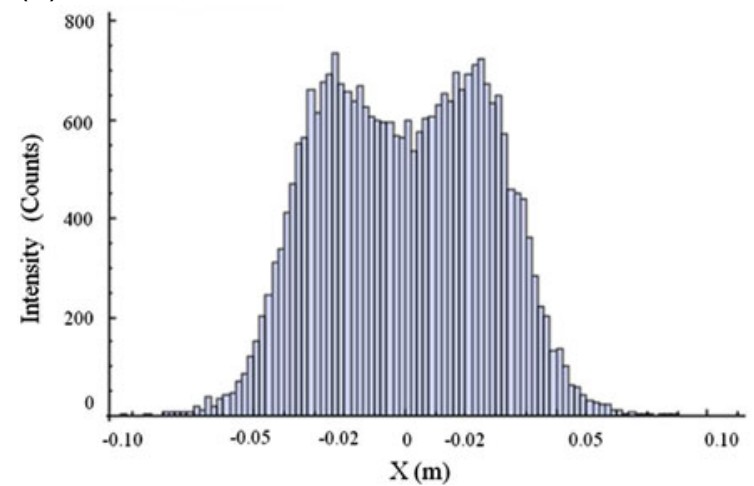

(b)

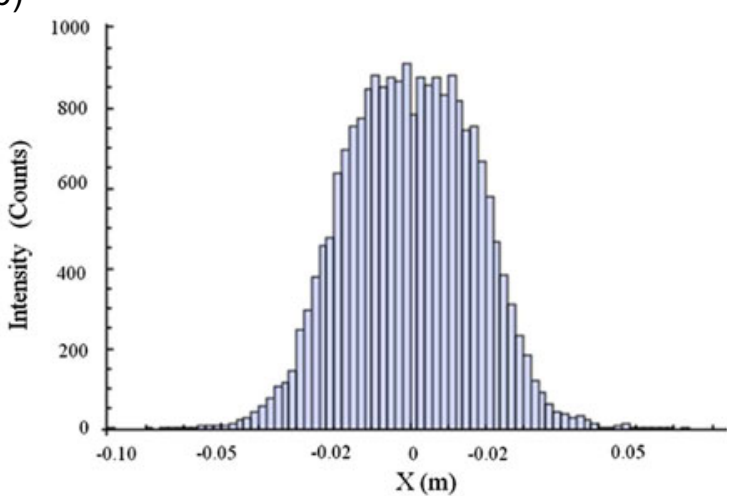

Fig. 11. (a) Numerical profile for $v_{\mathrm{F}}=61 \mathrm{~m} / \mathrm{s}$ with $P_{\mathrm{L}}=115 \mathrm{~mW}$ and $d_{\mathrm{L}}=16 \mathrm{~mm}$, (b) numerical profile for $v_{\mathrm{F}}=61 \mathrm{~m} / \mathrm{s}$ with $P_{\mathrm{L}}=15 \mathrm{~mW}$ and $d_{\mathrm{L}}=8 \mathrm{~mm}$.

move within the laser beam, centrifugal motions (making atoms leaving the centre) are more likely for low velocities and large numbers of absorbed photons. More generally, the role of the saturation parameter $s_{0}$, i.e., at resonance, the only parameter involved in the radiative force (Eq. (1)), is essential in the space-time dynamics of any slowed beam. As an example, Figure 11 shows radial profiles calculated with 2 different sets of laser beam parameters leading to different values of $s_{0}$, namely (a) laser power $P_{\mathrm{L}}=116 \mathrm{~mW}$, laser beam diameter $d_{\mathrm{L}}=$ $1.6 \mathrm{~cm}$ (at $z=z_{\mathrm{mir}}$ ), $s_{0}=14.30$, (b) $P_{\mathrm{L}}=15 \mathrm{~mW}$, $d_{\mathrm{L}}=0.8 \mathrm{~cm}, s_{0}=7.46$. In the former case, the depopulation reaches $18 \%$ of the total flux (instead of $10 \%$ in Fig. 10) whereas in the latter case this depopulation effect has completely disappeared. Present simulations have been obtained assuming an initial atomic beam strictly monokinetic and emitted by a point-like source. The more realistic case of an extended source (size $\sigma_{x}=$ $\left.\sigma_{y} \approx 1 \mathrm{~mm}\right)$ having a transverse velocity dispersion $\left(\sigma_{v} \approx\right.$ $1.1 \mathrm{~m} / \mathrm{s}$ ) has been also examined. These dispersions do not alter the present results by more than $5.3 \%$.

\section{Conclusion}

Experimental and numerical methods to investigate the slowing process of an atomic beam using a laser have been described. With some adjustments these methods can be generalized to other slowing techniques $[33,34]$ as well as to other species. The basic idea in this simulation is to model the atomic path as a random walk over a sequence of ten thousands of absorption - spontaneous emission cycles. This implies to determinate the distribution of random absorption times from known diffusion and emission rate constants.

Numerical determination of angular aperture and coherence radius of the slowed beam has been achieved using a 2D Monte Carlo imaging. This simulation appears to be a powerful method to determine these important parameters, some of them - as the coherence radius being difficult to be obtained experimentally. From available local densities, the feasibility of non-coherent atom optics experiments with slowed beams at "medium" velocity [35] can be demonstrated. The present calculations are made assuming the initial (supersonic) beam emitted from a point-like source, which represents the best accessible limit in terms of angular aperture after slowing. The very good agreement with experimental values obtained for this angular aperture indicates that the supersonic beam source is indeed point-like to a very good approximation. Moreover triggered imaging explains special effects exhibited by the experiment. Actually these effects, intrinsically linked to our experimental stet-up, consist of a de-population at the centre of the beam and correlatively the emergence of a partially slowed peak in the time-of-flight spectrum, two effects in which the saturation parameter plays a crucial role. The present numerical method has already given results of good quality ${ }^{1}$.

At a first sight, the conclusion about the use of simply slowed beams in scattering experiments at low velocities is pessimistic because of the wide angular spread and poor coherence radius. For example, to be used at a velocity of a few tens of $\mathrm{m} / \mathrm{s}$, such a beam needs to be strongly collimated or better focussed. Again a laser beam is an efficient tool for this purpose and its interaction with atoms could be accurately characterized by means of the present MC-code. Actually any configuration combining resonant light and atoms is relevant (provided that the semi-classical approximation is valid), in particular the use of a "pushing" laser to generate a slow atomic beam from a magneto-optical trap, a technique which has been successfully tested with metastable argon atoms [36].

Authors are members of the Institut Francilien de Recherche sur les Atomes Froids (IFRAF). They wish to warmly thank P. Pedri and L. Vernac from the Laboratoire de Physique des Lasers, University Paris 13, for stimulating discussions and their help for the numerical model. Many thanks are also due to T. Billeton from the same lab, who built the golden mirror, a key element of our experimental device.

1 The method has been successfully applied to a Zeeman deceleration of an Ytterbium beam and it is currently being extended to 2D and 3D magneto-optical traps. 


\section{References}

1. A. Ashkin, Science 210, 1081 (1980)

2. R. Blatt, W. Ertmer, P. Zoller, J. Hall, Phys. Rev. A 34, 3022 (1986)

3. V.I. Balikin, V.S. Letokhov, V.I. Mushin, JETP Lett. 29, 560 (1979); Sov. Phys. JETP 51, 692 (1980)

4. W. Phillips, H. Metcalf, Phys. Rev. Lett. 48, 596 (1982)

5. J.V. Prodan, W.D. Phillips, H. Metcalf, Phys. Rev. Lett. 49, 1149 (1982); J. Prodan, A. Migdall, I. So, H. Metcalf, J. Dalibard, Phys. Rev. Lett. 54, 992 (1985)

6. B.R. Mollow, Phys. Rev. A 12, 1919 (1975)

7. R. Maruyama, Ph.D. thesis, University of Washington, 2003

8. A. Aspect, J. Dalibard, A. Heidmann, C. Salomon, C. Cohen-Tannoudji, Phys. Rev. Lett. 57, 1688 (1986)

9. F. Shimizu, K. Shimizu, H. Takuma, Phys. Rev. A 39, 2758 (1989)

10. F. Lison, P. Schuh, D. Haubrich, D. Meschede, Phys. Rev. A 61, 013405 (1999)

11. T. Barrett, S.W. Dapore-Schwartz, M.D. Ray, G. Lafyatis, Phys. Rev. Lett. 67, 3484 (1991)

12. A. Molenaar, P. van der Straten, H.G.M. Heideman, Phys. Rev. A 55, 605 (1997)

13. J.G.C. Tempelaars, R.J.W. Stas, P.G. Sebel, H.C.W. Beijerinck, E.J.D. Vredenbregt, Eur. Phys. J. D 18, 113 (2002)

14. C. Slowe, L. Vernac, L. Vestergaard Hau, Rev. Sci. Instrum. 76, 103101 (2005)

15. J. Ramirez-Serrano, W. DeGraffenreid, J. Weiner, Phys. Rev. A 65, 052719 (2002)

16. J. Ramirez-Serrano, W. DeGraffenreid, J. Weiner, Phys. Rev. A 69, 042708 (2004)

17. M.D. Hoogerland, Ph.D. thesis, Technische Universiteit Eindhoven, 1993

18. G. Kistiakowsky, W. Slichter, Rev. Sci. Instrum. 22, 333 (1951)
19. R. Campargue, J. Phys. Chem. 88, 4466 (1984)

20. B. Brutschy, H. Haberland, J. Phys. E 10, 90 (1977)

21. S. Guberman, W. Goddard III, Phys. Rev. A 12, 1203 (1975)

22. J. Grucker, J. Baudon, F. Perales, G. Dutier, G. Vassilev, V. Bocvarski, M. Ducloy, J. Phys. B. 41, 1001 (2008)

23. Roentdek, MCP-delay line detector manual (2009), www. roentdek.com

24. M. Schellekens, Ph.D. thesis, University Paris XI, 2007

25. V.E. Lembessis, M. Babiker, C. Baxter, R. Loudon, Phys. Rev. A 48, 1594 (1993)

26. A.M. Abourabia, E. Hasseb, Chaos, Solitons and Fractals 12, 2295 (2001)

27. E.J.D. Vredenbregt, K. van Leeuwen, Am. J. Phys. 71, 760 (2003)

28. H.J. Metcalf, P. van der Straten, Optical Cooling and Trapping, revised edition (Springer-Verlag, New York, 2001)

29. R.J. Cook, Opt. Commun. 35, 347 (1980)

30. M. Hamamda, Ph.D. thesis, University Paris 13, 2011

31. M. Born, E. Wolf, Principles of Optics (Cambridge University Press, 1999)

32. J.-C. Karam, N. Wipf, J. Grucker, F. Perales, M. Boustimi, G. Vassilev, V. Bocvarski, C. Mainos, J. Baudon, J. Robert, J. Phys. B 38, 2691 (2005)

33. M. Hamamda, M. Boustimi, F. Correia, J. Baudon, T. Taillandier-Loize, G. Dutier, F. Perales, M. Ducloy, Phys. Rev. A 85, 023417 (2012)

34. J. Baudon, M. Hamamda, M. Boustimi, M. Bocvarski, T. Taillandier-Loize, G. Dutier, F. Perales, M. Ducloy, Nucl. Instrum. Methods B 279, 160 (2011)

35. M. Hamamda, M. Boustimi, V. Bocvarski, T. TaillandierLoize, G. Dutier, F. Perales, J. Baudon, M. Ducloy, Europhys. Lett. 98, 23001 (2012)

36. T. Taillandier-Loize, Ph.D. thesis, University Paris 13, 2014 